\title{
INTRODUZINDO CRITÉRIOS DE RISCO EM MODELOS DE CONTAMINAÇÃO PONTUAL PARA LOCAIS DE CARGA DE AGROTÓXICOS
}

LUCIANO GEBLER*

FLÁVIO BELLO FIALHO**

\begin{abstract}
O objetivo deste trabalho foi propor a adaptação do modelo de risco não pontual "Environmental Risk Index" (ERI), de Alister e Kogan (2006), mediante criação e aplicação de fatores de risco pontual derivados do manejo dos produtos nos locais de abastecimento de pulverizadores de agrotóxicos, gerando o modelo "índice de Risco pontual" (IRAp). O trabalho de campo foi executado nas dependências da Estação de Fruticultura de Clima Temperado (EFCT), da Embrapa Uva e Vinho, em Vacaria (RS), onde foram coletados os dados acerca da influência humana sobre o fator de risco ambiental do agrotóxico no momento do preenchimento do pulverizador. A fase de geração do modelo de simulação ocorreu na EFCT e na sede do centro da Embrapa Uva e Vinho, mediante adaptação do fator Dose (D) do ERI, transformando-o no Índice de Dose Pontual (iDp) e pela adequação matemática do fator Lixiviação (L) ao aproximar o comportamento dos 30 agrotóxicos teste expressos no modelo GUS para o modelo LIX. A introdução de critérios de risco envolvendo fatores humanos em casos de contaminação pontual por agrotóxicos provocou diferenças significativas em relação aos modelos destinados à avaliação de contaminação não pontual, e o Índice de Risco Ambiental pontual (IRAp) reproduziu os resultados de campo nas simulações. O IRAp demonstrou grande potencial para a análise preliminar ("screenning") dos riscos envolvidos nas repetidas operações de carga e limpeza de pulverizadores de agrotóxicos, permitindo o planejamento de longo prazo da escolha de produtos a serem utilizados na atividade, segundo a necessidade de segurança toxicológica do trabalhador e do ambiente ou o conjunto de ambas.
\end{abstract}

PALAVRAS-CHAVE: IMPACTOS AMBIENTAIS; SIMULAÇÃO DE RISCOS; ÍNDICE DE RISCO AMBIENTAL PONTUAL.

* Engenheiro Agrônomo, Doutor em Recursos Hídricos e Saneamento Ambiental, Embrapa Uva e Vinho, Vacaria, RS, Brasil (e-mail: lugebler@cnpuv.embrapa.br).

** Engenheiro Agrônomo, Ph.D. in Agricultural and Biological Engineering, Embrapa Uva e Vinho, Bento Gonçalves, RS, Brasil (e-mail: bello@cnpuv.embrap). 


\section{INTRODUÇÃo}

Os agroquímicos são ferramentas chaves dos sistemas produtivos modernos, representando, na maioria das vezes, a diferença entre o sucesso e o fracasso da safra agrícola. Os agrotóxicos produzem riscos ambientais e toxicológicos para o trabalhador e arredores pela produção de resíduos, manuseio indevido dos recipientes de armazenamento e derrames acidentais (SILVA et al., 2005)

$\mathrm{Na}$ área rural, o uso de agrotóxicos tem sido vinculado a problemas de contaminações não pontuais durante sua aplicação em culturas. Para reduzir esse tipo de impacto foram criadas formas de controlar resíduos, mediante monitoramento ou previsão de movimentação no perfil do solo usando modelagem matemática. Nos raros casos em que houve preocupação com a contaminação pontual, os modelos utilizados abrangiam os mesmos parâmetros previstos para avaliar contaminação difusa, ou seja, os parâmetros físicos, químicos e toxicológicos de testes padrões exigidos pelos órgãos de fiscalização dos Ministérios do Meio Ambiente e da Saúde. Há, entretanto, uma diferença fundamental entre contaminação pontual e difusa. Nos pontos de abastecimento de pulverizadores de agrotóxicos, o manuseio dos produtos ocorre de forma frequente e repetitiva, usando-se produtos comerciais concentrados que são diluídos em água para formar a calda de aplicação. $O$ risco de derrames aumenta com o número de cargas, que constitui indicador ambiental importante que não pode ser descartado numa análise de risco.

O conceito de risco pode ser descrito como o produto perigo $\times$ exposição, que implica na possibilidade de evento adverso ocorrer à alguém ou à alguma coisa (ALISTER e KOGAN, 2006). O processo de avaliação de riscos deve ser claramente estabelecido, usando-se diferentes graus de refinamento para sempre buscar a melhor representação possível da realidade (BILYARD et al., 1997). Indicadores ambientais podem ser usados para guiar respostas a eventos, sem quantificar o processo analisado. No entanto, perdem em eficiência e precisão para modelos matemáticos (BILYARD et al., 1997). Mesmo com essa limitação, o uso de indicadores provou ser eficaz e valioso pelo seu baixo custo operacional e resultados confiáveis. Pode ser aplicado até por usuários inexperientes, servindo como ferramenta de avaliação preliminar de risco. O conjunto de indicadores pode ser combinado para formar um índice resumido de atributos. Com base nessas premissas, buscou-se solução para modelar casos de contaminação de fontes pontuais envolvendo fatores humanos. O produto deste trabalho é resultado do aprimoramento do Environmental Risk Index (ERI) de Alister e Kogan (2006), modelo de uso simples, que oferece boas respostas de risco ambiental pelo uso de agrotóxicos em áreas agrícolas. As alterações foram sendo propostas de maneira construtiva (GEBLER, 2007) até sua estabilização, quando resultaram no Índice de Risco Ambiental pontual (IRAp).

O IRAp constitui indicador teórico/prático com base no ERI e nos critérios ambientais dos testes padrões do Conselho Nacional do Meio Ambiente (CONAMA) e da Agência Nacional de Vigilância Sanitária (ANVISA), exigidos para a liberação comercial de agrotóxicos no Brasil. Com o IRAp buscou-se reduzir a incerteza sobre o grau de risco desses produtos em rampas de abastecimento de pulverizadores de agrotóxicos (local com probabilidade particularmente alta de impacto ambiental e toxicológico). Nesses locais, grande variedade de agroquímicos comerciais concentrados é manuseada repetidamente. Isso pode afetar, em diferentes graus, os seres humanos envolvidos e os processos locais de atenuação ambiental natural.

O objetivo deste trabalho foi propor a adaptação do modelo de risco não pontual "Environmental Risk Index" (ERI), de Alister e Kogan (2006), mediante geração e aplicação de fatores de risco pontual, envolvendo o manejo nos locais de abastecimento de pulverizadores de agrotóxicos, gerando modelo de risco pontual "Índice de Risco de pontual” (IRAp).

\section{MATERIAL E METODOLOGIA DE ADAPTAÇÃO DO IRAp}

Para aplicar modelos e indicadores ambientais é necessário aproximar ou reduzir a realidade a número representativo de variáveis de estado, parâmetros e fatores que descrevam o 
ambiente de avaliação, mas suficientemente pequeno que permita o manuseio dos dados.

No IRAp são adotadas características físico-químicas dos agrotóxicos que refletem o pior cenário (maior lixiviação, menor coeficiente de partição octanol/água, $\mathrm{K}_{\text {ow’ }}$, maior persistência no solo, dentre outros), considerando-se esse procedimento como margem de segurança. Por isso, mesmo existindo piso com certo grau de impermeabilidade no local da carga, esse será considerado como solo sem impedimento da lixiviação.

O índice de risco ambiental original ERI (ALISTER e KOGAN, 2006) preve a introdução de parâmetros físico-químicos e ecotoxicológicos de agrotóxicos numa equação linear simples (Equação 1), cujos valores foram transformados em índice adimensional (FIGUEIRÊDO, 2008) a fim de permitir a correlação entre os fatores e avaliar qual dos produtos poderia apresentar maior risco ambiental em relação a outro:

$$
E R I=(P+L+V+T P) * D
$$

Em que:

$P=$ persistência no solo; $L=$ lixiviação; $V=$ volatilidade; $T P=$ perfil toxicológico; e $D=$ dose.

Cada um dos parâmetros constitui valor inteiro entre 1 (baixo) e 4 (muito alto), obtido de acordo com os intervalos listados na Tabela 1. O perfil toxicológico do agrotóxico (TP), obtido a partir de propriedades químicas e testes de campo fornecidas pelos fabricantes, órgãos ambientais ou fontes científicas será dado pela Equação 2:

$$
\mathrm{TP}=\mathrm{K}_{\mathrm{ow}}+\mathrm{Rfd}+\mathrm{LD}_{50}+\mathrm{AT}
$$

Em que:

$\mathrm{K}_{\mathrm{ow}}=$ coeficiente de partição octanol-água do agrotóxico; $\mathrm{Rfd}=$ dose de referência; $\mathrm{LD}_{50}=$ dose letal cutânea aguda para a população teste; $\mathrm{AT}$ = toxicologia animal.

Da mesma forma anterior, os parâmetros da Equação 2 são valores inteiros entre 1 e 4 , obtidos de acordo com a Tabela 1. Os dados para a execução do modelo podem ser obtidos de publicações científicas ou banco de dados específicos (WAUCHOPE et al., 1991; GEBLER et al., 2007).

\subsection{APRIMORAMENTOS DESTE TRABALHO}

Mantiveram-se os fatores de risco sugeridos por Alister \& Kogan (2006), com adaptações para atender os limites impostos pela legislação brasileira ou com base em testes experimentais a campo. O objetivo desses testes foi traduzir os parâmetros em fator único, porém com conhecimento das características ambientais do agrotóxico.

\subsubsection{Persistência no solo $(P)$}

A Persistência, representada pela meia vida no solo, permite aproximação de quanto tempo o agrotóxico levará para ser degradado no solo, com base na perda de $50 \%$ de sua massa inicial, conforme a Equação 3:

$$
t_{1 / 2}=\ln (2) / k
$$

Em que:

$t_{1 / 2}=$ meia vida em dias (baseada na conhecida equação de degradação de qualquer contaminante físico ou químico com cinética de primeira ordem) conforme Walker e Barnes 1981).

\subsubsection{Lixiviação (L)}

Fator que busca relacionar a possibilidade do agrotóxico lixiviar e a velocidade de descida no perfil do solo. Para tal utilizou-se o cálculo do Leacheability Index - LIX (SPADOTTO, 2002), 
adaptação do índice GUS desenvolvido por Gustafson (1989), com a vantagem de variar somente entre zero e um, enquanto que o GUS varia de $-\infty a+\infty$. O LIX é representado pela Equação 4:

Em que:

$$
\operatorname{LIX}=\exp ^{\left(-k^{*} \mathrm{Koc}\right)}
$$

$\mathrm{k}$ = constante de degradação de primeira ordem do agrotóxico $\left(\right.$ dia $\left.^{-1}\right)$ e $\mathrm{K}_{\mathrm{oc}}=$ coeficiente de adsorção ao carbono orgânico do solo ( $\mathrm{mg} \mathrm{C} / \mathrm{kg}$ ).

Os limites de cada nível dos LIX utilizados no IRAp foram calculados estatisticamente com base na diversidade e no volume de produtos utilizados neste trabalho, buscando-se a correlação mais apropriada com o GUS em relação ao artigo original (KOGAN et al., 2007). A relação entre os valores de GUS e LIX para o conjunto de 30 princípios ativos tem a forma de curva sigmóide, cujos limites assintóticos são 0 e 1. Tal relação pode ser expressa pela Equação 5 (FIALHO, 2002):

$$
\operatorname{LIX}=1 /\left(1+\exp \left(-8 \log 2^{*}(\text { GUS-xt }) / d x\right)\right)
$$

Em que:

xt e dx são parâmetros que representam, respectivamente, o valor de GUS correspondente ao valor de LIX de 0,5 e o intervalo de GUS em que a maior parte dos possíveis valores (88\%) de LIX está situada (o fator $8 \log 2$ é usado para corrigir a escala de $\mathrm{dx}$ ) de modo que a função se assemelhe a função de transição linear entre xt-dx/2 e xt+dx/2 (a integral definida entre - $\infty$ e xt é igual a dx/8 na função linear; o fator de correção faz com que a função de transição suave resulte no mesmo valor e, consequentemente, faça com que o fator $\mathrm{dx}$ expresse medida adequada do intervalo de transição).

Para a estimativa dos parâmetros xt e dx usou-se o software estatístico R (2009). A partir da equação resultante $\left(R^{2}=0,983\right)$, os limites de GUS tradicionalmente utilizados $(1,8$ e 2,8$)$ foram transformados em valores de LIX e estabelecidas as classes expressas na Tabela 1.

\subsubsection{Volatilização (V)}

A volatilização estabelece relação entre a degradação do agrotóxico no solo pela evaporação e sua remediação. Parte-se do princípio da diluição ambiental, segundo o qual quanto menor for a concentração no ponto de interesse, mais rapidamente o agrotóxico será eliminado. No ERI, o fator volatilização $(V)$ deriva da Equação 6:

$$
V=0,0029^{*} P^{*} M^{0,5}
$$

Em que:

$\mathrm{V}=$ taxa de perda do agrotóxico a campo; $\mathrm{P}=$ pressão de vapor; $\mathrm{e} \mathrm{M}=$ peso molecular, gerando ranking de 1 a 4 .

Como no IRAp, a volatilização passa a ser ferramenta para remediação naquele ponto. $O$ parâmetro é baseado diretamente na escala da pressão de vapor das substâncias, mantendo-se a relação de uma ordem de grandeza entre cada classe (Tabela 1).

\subsection{FATORES ADAPTADOS}

Os fatores dose (D) e perfil toxicológico (TP) sofreram modificações em razão do objetivo de desenvolver ferramenta para analisar a contaminação pontual no momento do carregamento do pulverizador e não a contaminação difusa. Alister e Kogan (2006) demonstraram a possibilidade de se alterar o ERI ao sugerirem a aplicação de índice alternativo, usado para águas subterrâneas, denominado $\mathrm{ERI}_{\text {loading }}$. Testaram a retirada do fator TP, considerado pelos autores originais como fator de medida de impacto ambiental. 
Na equação no IRAp, o TP passou a ser considerado como fator de risco toxicológico direto ao operador do ponto de abastecimento, sem contar a poluição difusa. Além disso, no IRAp foi alterado o fator dose (D), acrescentando-se os parâmetros NC e CT (Número de Carregamentos e Classe Toxicológica). Isto permitiu ao modelo representar o risco de derrames, potencializado pela repetibilidade da operação de carregamento do pulverizador sempre no mesmo local.

\subsubsection{Dose (D)}

A dose aplicada em determinada área, normalmente uma única vez por período, determina o incremento do risco a ser considerado. No desenvolvimento do IRAp, considerando a recorrência da natureza da atividade de risco, propõe-se associar dois sub-fatores relacionados com o incremento do risco de acidentes naquele ponto. O sub-fator NC deriva do incremento estatístico de ocorrer pelo menos um derrame à medida que o número de operações de mistura de calda aumenta no ponto de abastecimento do pulverizador para cumprir uma sessão de aplicação. Isto ocorre independente da área total a ser tratada de forma semelhante ao IRA, cujo fator Dose se refere à concentração do princípio ativo em um hectare somente. Além disso, foi adotado subfator de risco toxicológico como segurança ao operador daquele ponto, CT, utilizando-se a classe toxicológica do produto como indicador do nível de segurança humana em relação à operação. Isto se deve ao conceito de que há menor risco ao se tratar com maiores volumes de agrotóxicos menos tóxicos do que com pequenos volumes muito tóxicos. Esse conceito foi avaliado nas dependências da Estação Experimental de Fruticultura Temperada, em Vacaria (RS), sendo mensurado em sessões de aplicação de produtos antes e depois da execução de treinamentos aos operadores das máquinas agrícolas, demonstrando que a possibilidade de acidentes aumenta com o decorrer do tempo.

Os dados obtidos são reforçados por Roettele (2008), que apontou o treinamento como fator de maior influência na segurança do manejo de agrotóxicos. No caso de Vacaria, verificouse que após o treinamento houve redução de volume de calda e resíduos derrubados no piso do abastecedor da ordem de 10 vezes, com diminuição da média de 199,5 litros de líquido coletado resultante de uma sessão de aplicação para a média de 21,33 litros (3 repetições). Essa mesma ordem de grandeza é encontrada entre uma categoria e outra de classes toxicológicas para agrotóxicos adotadas pelo Ministério da Saúde do Brasil.

As adaptações em $\mathrm{D}$ resultam no índice de dose pontual (iDp), conforme a Equação 7:

$$
i \mathrm{Dp}=\mathrm{D}+\left(\mathrm{NC} \mathrm{C}^{*} \exp ^{(-\mathrm{CT})}\right)
$$

\subsubsection{Toxicologia}

O perfil toxicológico (TP) significa o fator de segurança que o agrotóxico representa ao operador (visto como parte do ambiente). O sub-fator AT presente no IRA pode ser desconsiderado, pois se busca o nível de risco causado pelo contato recorrente direto com o agrotóxico pelo manejo. Também, o parâmetro Rfd (dose de referência) foi substituído pelo valor da Ingestão Diária Aceitável (IDA), representando o risco apresentado pelo agrotóxico ao ser ingerido (no caso da ingestão acidental por contato com a boca ou partículas de spray). Desta forma, o fator TP do IRAp passa a apresentar a seguinte forma:

$$
\mathrm{TP}=\mathrm{K}_{\mathrm{oW}}+\mathrm{IDA}+\mathrm{CL}_{50}
$$

Em que:

TP = Perfil Toxicológico; IDA = Ingestão Diária Aceitável; $\mathrm{CL}_{50}=$ Dose inalatória letal para $50 \%$ da população teste; $\mathrm{K}_{\mathrm{ow}}=$ Coeficiente de partição octanol-água, responsável por indicar o potencial 
de bioconcentração que o agrotóxico pode oferecer ao trabalhador (BRIGGS, 1981; ISNARD e LAMBERT, 1988), uma vez que o log do $\mathrm{K}_{\text {ow }}$ constitui fator diretamente correlacionado com 0 potencial de acumulação nos ácidos graxos dos seres vivos.

Substâncias com valores de log acima de 2,0 requerem maiores cuidados no manejo e acima de log 3,0 passam a ser consideradas como alto risco ambiental e toxicológico (PARAIBA e SAITO, 2005).

Além dos dados físico-químicos dos princípios ativos, é necessário obter dados relacionados com o agrotóxico ou com o manejo porque o usuário fará emprego de produtos comerciais.

\section{INFORMAÇÕES PARA APLICAÇÃO DO IRAp}

As informações necessárias para o uso do IRAp, além dos dados físico-químicos são:

- Concentração do principio ativo no produto formulado comercial - devido à existência de diversas formulações comerciais do mesmo princípio ativo, com concentrações diferentes, afetando principalmente o fator Dose;

- Classe toxicológica - o mesmo ingrediente ativo pode apresentar diferentes classes toxicológicas por questões de concentração no formulado comercial, ou relacionado aos demais componentes do agrotóxico;

- Número de carregamentos de agrotóxicos - normalmente é necessário mais de um procedimento de enchimento de tanque de pulverizador para a execução do serviço que, na maioria das vezes, abrange mais do que um hectare, afetando o grau de risco de acidentes, também representado no fator Dose.

Mantendo a equação final na forma linear, estabeleceu-se a nova conformação do IRAp:

$$
\text { IRAp }=(P+L+V+T P) * i D p
$$

\section{FORMA DE APLICAÇÃO DO IRAp}

O IRAp foi desenvolvido para ser aplicado diretamente pelo usuário final a partir de planilhas de cálculo, na forma de preenchimento de cada parâmetro ou mediante banco de dados pré-definidos para informações fixas referentes aos agrotóxicos. A variável que sempre fica a encargo do usuário é a definição do número de carregamentos para cada sessão de aplicação de produto, que sozinha pode alterar o escore final da análise.

A avaliação de risco ambiental feita pelo índice pode exigir parâmetros fixos para possibilitar a comparação entre os dados. Muitas vezes isso não é possível, principalmente em casos com variação da concentração por hectare do mesmo princípio ativo sob análise, afetando o número de carregamentos e se refletindo no fator iDp. Isso pode contribuir para a alteração do fator final de risco. Comparando-se os valores do IRAp entre o Malathion 1000CE (Dose máxima $1000 \mathrm{~g} / \mathrm{L}$.ha) e o Malathion 500CE (Dose máxima $750 \mathrm{~g} / \mathrm{L}$.ha) verifica-se que seriam necessárias somente 7,5 cargas do Malathion $1000 \mathrm{CE}$ para se igualar à concentração de ingrediente ativo por hectare de 10 cargas do Malathion 500CE. Porém, caso seja mantido o mesmo número de cargas para ambos durante a simulação, pode haver alteração de categoria no resultado final da avaliação.

Adotou-se da literatura (ALISTER \& KOGAN, 2006) que os fatores para cada agrotóxico sejam classificados como de baixo (1), médio (2), alto (3) ou muito alto risco (4), gerando no final da equação número inteiro entre 4 e 64, que representa o nível de risco estimado, conforme a Tabela 1. 


\section{TABELA 1- TABELA DE CLASSIFICAÇÃO DE PARÂMETROS HUMANOS, FÍSICO-QUÍMICOS E ECOTOXICOLÓGICOS PARA APLICAÇÃO NO ÍNDICE DE RISCO AMBIENTAL PONTUAL - IRAp}

\begin{tabular}{|c|c|c|c|c|c|c|c|c|c|c|}
\hline Tipo & \multirow{2}{*}{$\begin{array}{c}P \\
\left(t_{1 / 2}, \text { dias }\right)\end{array}$} & \multirow{2}{*}{$\begin{array}{l}\mathrm{L} \\
(\mathrm{LIX})\end{array}$} & \multirow{2}{*}{$\begin{array}{c}\mathrm{V} \\
\text { (Pascal) }\end{array}$} & \multirow{2}{*}{$\begin{array}{l}\mathrm{D} \\
\mathrm{kg}\end{array}$} & \multirow{2}{*}{ iDp } & \multirow{2}{*}{ TP } & \multirow{2}{*}{$\begin{array}{c}\text { IDA } \\
\text { (mg/kg p.v.) }\end{array}$} & \multirow{2}{*}{$\mathrm{K}_{\mathrm{ow}}$} & \multirow{2}{*}{$\begin{array}{c}\mathrm{CL}_{50} \\
(\mathrm{mg} / \mathrm{kg} \\
\text { p.v.) }\end{array}$} & \multirow{2}{*}{ IRAp } \\
\hline $\begin{array}{l}\text { Nível de } \\
\text { risco }\end{array}$ & & & & & & & & & & \\
\hline 1 Baixo & $<30$ & 0 & $<0,00133$ & $\leq 1$ & $\leq 1$ & 3 & $>0,1$ & $<1$ & $>4000$ & 4 a 8 \\
\hline 2 Médio & 30 a 60 & $>0$ a 0,025 & 0,00133 a 0,0133 & 1 a 2 & 1 a 2 & 3 a 6 & 0,1 a 0,01 & 1 a 2 & 4000 a 400 & 9 a 24 \\
\hline 3 Alto & 60 a 90 & 0,025 a 0,135 & 0,0133 a 0,133 & 2 a 3 & 2 a 3 & 6 a 9 & 0,01 a 0,001 & 2 a 3 & 400 a 40 & 25 a 48 \\
\hline 4 Muito alto & $>90$ & $>0,135$ & $>0,133$ & $\geq 3$ & $\geq 3$ & $>9$ & $<0,001$ & $>3$ & $<40$ & $>48$ \\
\hline
\end{tabular}

$\mathrm{P}=$ persistência no solo; $\mathrm{t}_{1 / 2}=$ meia vida no solo; $\mathrm{L}=$ lixiviação; $\mathrm{V}$ = volatilização; $\mathrm{D}=$ dose; TP = perfil toxicológico; IDA = ingestão diária aceitável; $\mathrm{K}_{\mathrm{OW}}=$ Coeficiente de partição octanol-água; $\mathrm{CL}_{50}=$ Dose inalatória letal para $50 \%$ da população teste; IRAp = índice de risco ambiental pontual.

Para exemplificar o uso do modelo foram testados 30 ingredientes ativos de agrotóxicos, distribuídos em 67 formulações comerciais usadas na agricultura brasileira (Tabela 2). Com base no trabalho de Gebler, Pelizza e Almeida (2007) e para facilitar a aplicação do modelo foi estabelecido seu uso em planilha eletrônica (Figura 1) que pode ser adaptada pelo usuário.

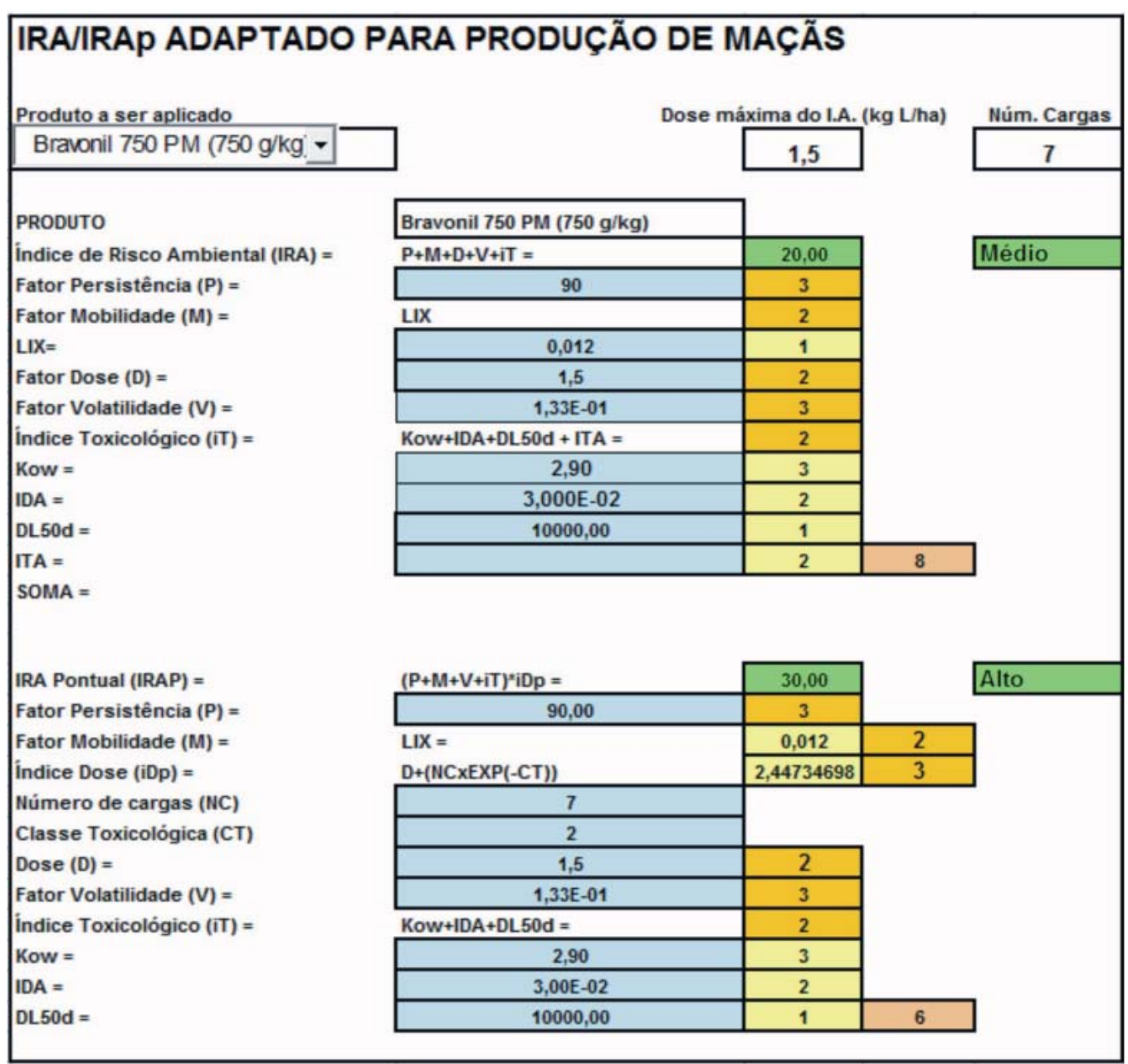




\section{TABELA 2 - RELAÇÃO DOS PRINCÍPIOS ATIVOS QUE SERVIRAM DE BASE PARA ESTABELECIMENTO DOS PARÂMETROS DO IRAp}

\begin{tabular}{|c|c|c|c|c|c|c|c|}
\hline PRINCÍPIO ATIVO & CT & PV & KOW & KOC & $1 / 2 \mathrm{~V}$ & IDA & CL50 \\
\hline BENOMIL & 3 & 1,93E-08 & 3,11 & 3,28 & 365 & $1,000 \mathrm{E}-01$ & $>10000$ \\
\hline CAPTANA & 3 & 1,20E-02 & 3,40 & 1,52 & 60 & $1,000 \mathrm{E}-01$ & $>4500$ \\
\hline CARBENDAZIM & 3 & $9,00 \mathrm{E}-02$ & 1,77 & 2,3 & 150 & $1,000 \mathrm{E}-02$ & $>2000$ \\
\hline CLOROTALONIL & 1 & 1,33E-01 & 2,90 & 2,76 & 90 & $3,000 \mathrm{E}-02$ & $>10000$ \\
\hline CIPRODINIL & 3 & $5,00 \mathrm{E}-05$ & 4,00 & 3,56 & 150 & 3,750 E-02 & $>2000$ \\
\hline DODINA & 1 & 1,30E-02 & 1,15 & 4,00 & 20 & $1,000 \mathrm{E}-02$ & $>6000$ \\
\hline FENARIMOL & 2 & $3,00 \mathrm{E}-02$ & 3,96 & 1,08 & 365 & 1,000E-02 & $>2000$ \\
\hline FLUAZINAM & 2 & 1,10E-03 & 3,56 & 3,23 & 49 & $1,100 \mathrm{E}-02$ & $>2000$ \\
\hline FOSETIL & 4 & 1,00E-03 & $-2,53$ & 2,22 & 0,0416 & $3,000 E+00$ & $>3200$ \\
\hline IPRODIONA & 4 & 1,01E-03 & 3,10 & 2,82 & 60 & $4,000 \mathrm{E}-02$ & 2500 \\
\hline KRESOXIM-METIL & 3 & 2,30E-06 & 3,40 & 3,23 & 1 & $4,000 \mathrm{E}-01$ & $>2000$ \\
\hline MANCOZEBE & 3 & 1,70E-05 & 1,33 & 3,77 & 139 & $3,000 \mathrm{E}-02$ & $>10000$ \\
\hline MICLOBUTANIL & 1 & $2,13 E-04$ & 2,94 & 2,70 & 71 & $3,000 \mathrm{E}-02$ & 2290 \\
\hline PIRIMETANIL & 3 & $2,30 E+03$ & 2,84 & 2,92 & 27 & $2,000 \mathrm{E}-01$ & $>5000$ \\
\hline TETRACONAZOLE & 2 & 1,60E-03 & 3,53 & 2,73 & 403 & $1,000 \mathrm{E}-01$ & $>2000$ \\
\hline $\begin{array}{l}\text { TIOFANATO } \\
\text { METÍLICO }\end{array}$ & 4 & 1,33E-05 & 1,50 & 0,08 & 10 & $8,000 \mathrm{E}-02$ & $>10000$ \\
\hline TIABENDAZOL & 4 & 5,32E-04 & 2,47 & 3,24 & 1444 & $1,000 \mathrm{E}-01$ & $>5000$ \\
\hline TRIFORINA & 2 & $2,70 \mathrm{E}-01$ & 2,20 & 2,30 & 21 & $2,000 \mathrm{E}-02$ & $>10000$ \\
\hline ABAMECTINA & 3 & $2,00 \mathrm{E}-04$ & 3,99 & 3,60 & 56 & $2,000 \mathrm{E}-03$ & $>330$ \\
\hline CLORPIRIFÓS & 2 & 1,04E-01 & 5,27 & 2,92 & 365 & $1,000 \mathrm{E}-02$ & $>2000$ \\
\hline DIAZINONA & 2 & $2,00 \mathrm{E}-02$ & 3,81 & 2,12 & 90 & $2,000 \mathrm{E}-03$ & 3600 \\
\hline DICOFOL & 1 & 1,33E-03 & 4,28 & 3,70 & 68 & $2,000 \mathrm{E}-03$ & 5000 \\
\hline DIMETOATO & 1 & $8,50 \mathrm{E}-03$ & 2,71 & 0,13 & 122 & 2,000E-03 & 600 \\
\hline FENITROTIONA & 2 & $1,80 \mathrm{E}-02$ & 3,43 & 2,63 & 42 & $3,000 \mathrm{E}-03$ & 890 \\
\hline METIDATIONA & 2 & 1,86E-01 & 4,72 & 0,84 & 23 & 1,000E-03 & 94 \\
\hline TEBUFENOZIDA & 4 & 9,99E-06 & 4,25 & 4,54 & 115 & $2,000 \mathrm{E}-02$ & $>5000$ \\
\hline CARBARIL & 2 & $2,80 \mathrm{E}-03$ & 3,46 & 1,41 & 100 & $3,000 \mathrm{E}-03$ & $>2000$ \\
\hline GLUFOSINATO & 3 & $1,00 \mathrm{E}-04$ & 0,10 & 3,09 & 40 & $2,000 \mathrm{E}-02$ & $>2000$ \\
\hline GLIFOSATO & 4 & 1,00E-03 & 1,67 & $-0,43$ & 174 & $3,000 \mathrm{E}-01$ & $>2000$ \\
\hline SIMAZINA & 3 & $5.00 \mathrm{E}-03$ & 2.30 & 1.43 & 186 & $5.000 \mathrm{E}-03$ & 3100 \\
\hline
\end{tabular}

$\mathrm{CT}$ = classe toxicológica; $\mathrm{PV}=$ pressão de vapor; $\mathrm{KOW}=$ constante de partição octanol/água; $\mathrm{KOC}=$ constante de partição octanol/carbono orgânico; $1 / 2 \mathrm{~V}$ = meia vida do produto em água; IDA = ingestão diária aceitável; CL50 = índice toxicológico de contaminação dermal para $50 \%$ da população.

\section{RESULTADOS E DISCUSSÃO}

O objetivo do IRAp de quantificar os riscos em áreas cuja contaminação ocorre pontualmente (concentrada), o torna diferente do objeto do estudo do IRA. Espera-se que haja diferenciação numérica entre os resultados dos dois modelos para alguns produtos, demonstrando que há agrotóxicos potencialmente mais perigosos em uso na forma difusa, enquanto que em outros o risco maior está no uso pontual. A aplicação desse índice proporcionará incremento na segurança da operação de carregamento, conforme agrotóxicos distintos sejam preparados nas rampas. A deposição de produto não é aceita como prática normal em ponto de abastecimento de pulverizadores, porém por deficiências de equipamento e de manejo ocorrem desde pequenos gotejamentos até grandes derrames. Individualmente, utilizando-se a dose prescrita para a máxima eficiência agronômica de cada produto e fixando-se o número de cargas em 10 (para fins dessa simulação como exemplo), o IRAp indicou níveis mais críticos em relação ao ERI quando os produtos apresentaram classe toxicológica de maior risco (1 ou 2), ou diferentes concentrações do princípio 
ativo no produto comercial, chegando a resultar em níveis de risco distintos entre os resultados (sempre mais baixos no ERI). Com o IRAp, o produtor pode decidir antecipadamente se aplica métodos de prevenção aos riscos (barreiras físicas, químicas, compostagem, redução da toxicidade dos produtos, redução do número de cargas, treinamento, dentre outros), ou terá a indicação do possível grau de mitigação posterior (se necessário).

Ao analisar os resultados da aplicação do IRA aos princípios ativos glifosato (herbicida), dimetoato (inseticida) e clorotalonil (fungicida), comumente empregados em diversos cultivos, foram obtidos respectivamente os valores 33/alto, 13/médio e 20/médio (Tabela 3). Esses valores levam em conta somente o risco ao ambiente pela contaminação difusa. Com a aplicação do IRAp, os valores passaram para 44/alto (glifosato), 52/muito alto (dimetoato) e 30/alto (clorotalonil). Apesar de no caso do glifosato a classificação permanecer a mesma, numericamente houve alteração do valor em 11 pontos, recomendando maior cuidado no manuseio e permitindo ao usuário diferenciar riscos durante as etapas de uso. Já para o caso do dimetoato, houve alteração de valor de 13 para 52 , e de classe de médio para muito alto, alertando ao usuário que o risco pontual desse produto é muitas vezes maior do que o risco difuso, principalmente quanto à toxicologia. Esse indicador aponta a necessidade de maior investimento em segurança no ponto de abastecimento de pulverizadores em que ocorre o manuseio do produto. O clorotalonil também apresentou incremento numérico de 10 pontos, que foi suficiente para sua mudança de classe, demonstrando também ao usuário o risco toxicológico existente pelo uso do produto de maneira incorreta na forma concentrada.

\section{TABELA 3 - AVALIAÇÃO DO ÍNDICE DE RISCO AMBIENTAL PONTUAL (IRAP) DE TRÊS PRINCÍPIOS ATIVOS}

\begin{tabular}{lcccc}
\hline Marca comercial & Princípio Ativo & $\begin{array}{c}\text { Classificação de } \\
\text { uso }\end{array}$ & $\begin{array}{c}\text { Classificação IRA } \\
\text { Valor/classe }\end{array}$ & $\begin{array}{c}\text { Classificação IRAp } \\
\text { Valor/classe }\end{array}$ \\
\hline Agrisato 480 CS & Glifosato & Herbicida & 33/Alto & 44/Alto \\
Tiomet 400CE & Dimetoato & Inseticida & 13/Médio & 52/Muito Alto \\
Bravonil 750PM/ & Clorotalonil & Fungicida & 20/Médio & 30/Alto \\
\hline
\end{tabular}

\section{CONCLUSÃO}

A introdução de critérios de risco envolvendo fatores humanos em casos de contaminação pontual por agrotóxicos provoca diferenças significativas em relação aos modelos destinados à avaliação de contaminação não pontual.

O Índice de Risco Ambiental pontual reproduz os resultados de campo nas simulações, apresenta fácil manuseio em planilhas eletrônicas e clareza de resultado mediante escala numérica direta.

OIRAp demonstrou grande potencial na análise preliminar ("screenning") dos riscos envolvidos nas repetidas operações de carga e limpeza de pulverizadores de agrotóxicos, permitindo o planejamento de longo prazo na escolha de produtos a serem utilizados na atividade, segundo a necessidade de segurança do ambiente, de segurança toxicológica ao trabalhador, ou o conjunto de ambas.

\section{ABSTRACT \\ INTRODUCING RISK CRITERIA INTO MODELS OF POINT SOURCE CONTAMINATION IN PESTICIDE LOAD PADS}

The objective of this work was to propose an adaptation of the non-punctual Environmental Risk Index (ERI) of Alister \& Kogan (2006), through generation and application of punctual risk factors derived from the management of products in agrochemical equipment loading places, creating a model of point source environmental risk index (IRAp). Field work was conducted at the Embrapa's Grape and Wine Temperate Climate Fruit Research 
Station (EFCT), at Vacaria (RS - Brazil) where data about human influence on the environmental risk factor of the agrochemical were collected during equipment loading moment. Model generation involved the adaptation of the dose factor (D) of ERI, transforming it into a punctual dose index (iDp), and the mathematical adjustment of the leaching factor (L), by relating the behavior of the GUS and LIX models of 30 tested agrochemicals. The introduction of risk criteria involving human factors in cases of punctual contamination by agrochemicals presented significant differences in comparison to non-punctual contamination models, and point source environmental risk index (IRAp) was able to reproduce field results on the simulations. IRAp proved to be a tool of great potential in screening of risks involved in repeated loading and cleaning operations of agrochemical spraying equipment, allowing long term planning of products choice, along with environmental safety needs, worker safety requirements, or both.

\section{KEY-WORDS: ENVIRONMENTAL IMPACT; RISK SIMULATION; POINT SOURCE ENVIRONMENTAL RISK INDEX.}

\section{REFERÊNCIAS}

1 ALISTER, C.; KOGAN, M. ERI: Environmental risk index. A simple proposal to select agrochemicals for agriculture use. Crop protection, v. 25, p. $202-211,2006$.

2 BILYARD, G. R.; BECKERT, H.; BASCIETTO, J. J.; ABRAHAMS, C. W.; DYER, S. A.; HASELOW, L. A. Using the data quality objectives process during the design and conduct of ecological risk assessment. Washington, D.C.: U.S. Department of Energy, 1997. 124 p.

3 BRIGGS, G. Theoretical and experimental relationships between soil adsorption, octanol-water partition coefficients, water solubilities, bioconcentration factors. Journal of Agriculture and Food Chemistry, v. 20, p.1050-1059, 1981.

4 FIALHO, F. B. Funções de transição para uso em modelagem. Concórdia, SC: Embrapa Suínos e Aves, 2002.5 p. (Comunicado Técnico, 310).

5 FIGUEIRÊDO, M. C. B. DE. Modelo de avaliação de desempenho ambiental de inovações tecnológicas agroindustriais considerando o conceito de ciclo de vida e a vulnerabilidade ambiental: ambitec-ciclo de vida. 2008. 424 p. Tese (Doutorado em Engenharia Civil), Universidade Federal do Ceará, Fortaleza, 2008.

6 GEBLER, L. Índice de risco ambiental para pontos de abastecimento de pulverizadores de agrotóxicos (IRAp), uma adaptação do environmental risk index (ERI) para a produção integrada. In: SEMINÁRIO BRASILEIRO SOBRE PRODUÇÃO INTEGRADA DE FRUTAS, 9., e SEMINÁRIO SOBRE O SISTEMA AGROPECUÁRIO DE PRODUÇÃO INTEGRADA, 1., 2007, Bento Gonçalves. Resumos... Bento Gonçalves: Embrapa Uva e Vinho, 2007. p. $221-225$.

7 GEBLER, L.; PELIZZA, T. R.; ALMEIDA, D. L. de. Variáveis ambientais e toxicológicas de agroquímicos utilizados na produção integrada de maçãs visando modelagem matemática. Revista de Ciências Agroveterinárias, v.5, p.169-184, 2007.

8 GUSTAFSON, D. I. Groundwater ubiquity score: a simple method for assessing agrochemical leachability. Environmental Toxicology Chemistry, v. 58, p. 339-357, 1989.

9 ISNARD, P.; LAMBERT, S. Estimating bioconcentration factors from octanol-water partition coefficient and aqueous solubility. Chemosphere, v. 17, p. 21-34, 1988.

10 KOGAN, M; ROJAS, S.; SUÁREZ, F.; MUNÕZ, J. F.; ALISTER, C. Evaluation of six pesticide leaching indexes using field data of herbicide application in Casablanca Valley, Chile. Water Science \& Technology, v. 56, n.2, p.169-178, 2007.

11 PARAIBA, L. C.; SAITO, M. L. Modelagem da distribuição ambiental de poluentes orgânicos encontrados em biossólidos. Pesquisa Agropecuária Brasileira, Brasília, v. 40, n. 9, p.853-860, 2005.

12 R DEVELOPMENT CORE TEAM. R: a language and environment for statistical computing. Vienna, Austria: R Foundation for Statistical Computing, 2009.Available at: http://www.R-project.org. Access on: Mar. 15th, 2011.

13 ROETTELE, M. Strategies to reduce point source losses of ppp to water focus on "behaviour, technique and infrastructure": results and lessons learned from the Topps - project. Aspects of Applied Biology, n. 84, p.357-368, 2008.

14 SPADOTTO, C. A. Screening method for assessing pesticide leaching potential. Pesticidas: revista de ecotoxicologia e meio ambiente, Curitiba, v.12, p. 69-78, 2002.

15 SILVA, J. M. da; NOVATO-SILVA, E.; FARIA, H. P.; PINHEIRO, T. M. M. Agrotóxico e trabalho: uma combinação perigosa para a saúde do trabalhador rural. Ciênc. Saúde Coletiva, v.10, n.4. p.891-903, 2005.

16 WALKER, A.; BARNES, A. Simulation of herbicide persistence in soil: a revised computer model. Pesticide Science, Chichester, v.12, p.123-132, 1981.

17 WAUCHOPE, R. D.; BUTTLER, T. M.; HORNSBY, A. G.; AUGUSTIJN-BECKERS, P. W. M.; BURT, J. P. The SCS/ARS/ CES pesticide properties database for environmental decision-making. Environmental Contamination Toxicology, v.123. p.1-36, 1991. 